\title{
Portafolio de inversiones y desempeño del sistema privado de pensiones peruano: 2000 - 2005*
}

David Juan Sanchez Cruz

\section{RESUMEN}

El presente trabajo es una visión de la administración financiera y gestión de portafolio del Sistema Privado de Pensiones (SPP) en el Perú, que toma como insumos para los análisis a realizar, las cifras que publica el supervisor oficial. En busca de la objetividad, nos remitimos a la metodología financiera, las fuentes oficiales y los resultados de ellos obtenidos.

Como marco teórico se tiene a la Teoría de Mercados Eficientes, la Teoría del Portafolio, el Modelo de Valuación de Activos de Capital (CAPM), los índices de Sharpe, Treynor, Jensen y Treynor-Mazuy.

Palabras clave: Sistema de Pensiones, activos financieros, portafolio, desempeño.

\section{ABSTRACT}

The present work is a vision of financial administration and portfolio management of Peruvian Private Pensions System (SPP), which takes like consumptions for the analyses to realize, the data that publishes the official supervisor. In search of the objectivity, we were sent, to the financial methodology, the official sources and the obtained results.

* Resumen de Tesis: David Juan Sanchez Cruz "Portafolio de inversiones y desempeño del sistema privado de pensiones peruano; 2000 - 2006” UNMSM. Biblioteca de Ciencias Económicas. 2007 


\section{David Juan Sanchez Cruz}

We take as Theoretical Frame, the theory of Efficient Markets, the theory of the Portfolio, the Capital Assets Pricing Model (CAPM), the index Sharpe, Treynor, Jensen and Treynor-Mazuy.

Keywords: Financial system of pensions, assets, portfolio, performance.

\section{INTRODUCCIÓN}

Los principales inversionistas institucionales (los fondos mutuos, fondos de inversión y el Sistema Privado de Pensiones (SPP), siendo este último nuestro objeto de estudio) cumplen un rol fundamental en el mercado de capitales peruano, al ser los principales demandantes de activos financieros. La participación de estos inversionistas institucionales provee de recursos financieros a diversos sectores de la economía, lo que en última instancia se observa en un mayor crecimiento económico. El mercado de capitales peruano experimenta un notable desarrollo desde la década de los 90, alcanzando en el 2006 cifras record.

Para evaluar el desempeño de las Administradoras de Fondos de Pensiones (AFP), estas deben estar ajustadas por riesgo. Entonces, surgen las interrogantes ¿Qué entendemos por riesgo?, ¿cómo lo cuantificamos? y ¿̇e qué tipo de riesgo estamos hablando? Preguntas que requieren una respuesta a la hora de valorizar activos.

Tomo como punto de referencia, estudios realizados con anterioridad por renombrados investigadores peruanos, tales como los trabajos de: Paúl Castillo y Ruy Lama (1998). Evaluación de portafolio de los inversionistas institucionales: fondos mutuos y fondos de pensiones. BCRP; Eduardo Morón Pastor, Eliana Carranza Noguera, Rudy Loo-Kung Agüero (2004). Teoría de Mercados Oligopólicos y Economías de Escala. Lecciones para el caso de las AFP. CIUP.

\section{EL SISTEMA PRIVADO DE PENSIONES}

El desarrollo del $\mathrm{SPP}^{1}$ ha creado fuentes alternativas de financiamiento para diversos sectores de la economía incrementando la competencia con la banca. El fondo de pensiones acumulado por el SPP ha hecho posible financiar proyectos de inversión en

1 Mayor información del SPP, podemos obtenerla en la página de la Superintendencia de Banca Seguros y AFP. 
Portafolio de inversiones y desempeño del sistema privado de pensiones peruano: 2000 - 2005

varios sectores económicos. La inversión en estos sectores se realiza adquiriendo una amplia variedad de instrumentos financieros.

Cuadro N. ${ }^{\circ}$ 1. Activos en el Mercado Local

\begin{tabular}{lll}
\hline \multicolumn{1}{c}{ Tipo de Instrumento } & \multicolumn{1}{c}{$\begin{array}{c}\text { Emisiones } \\
\text { Millones de US\$ }\end{array}$} & \multicolumn{1}{c}{$\begin{array}{c}\text { Emisiones } \\
\text { Millones de S/. }\end{array}$} \\
\hline A. SECTOR GOBIERNO & 416,3 & $10.246,5$ \\
1. Bonos Soberanos & & $10.227,1$ \\
1.1 Tasa Fija & & $8.247,4$ \\
1.2 VAC & 416,3 & $1.979,7$ \\
2. Bonos Específicos & 0,0 & 19,4 \\
B. EMISIONES BCRP & & $6.321,3$ \\
1. Certificados de Depósito BCRP & $2.767,1$ & $6.321,3$ \\
C. SECTOR PRIVADO*/ & 410,0 & $5.419,7$ \\
1. Bonos de Arrendamiento Financiero & 53,9 \\
2. Bonos Corporativos & $1.478,9$ & $4.452,2$ \\
3. Instrumentos de corto plazo & 58,6 & 70,0 \\
4. Bonos Subordinados & 187,3 & 248,0 \\
5. Bonos de Titulización & 536,1 & 114,7 \\
6. Certificados de Depósito Negociables & 10,0 & 480,9 \\
7. Bonos Hipotecarios & 86,2 & 0,0 \\
TOTAL GENERAL ( A+B+C ) & $\mathbf{3 . 1 8 3 , 4}$ & $\mathbf{2 1 . 9 8 7 , 5}$ \\
\hline
\end{tabular}

Fuente: CONASEV.

Gráfico N. ${ }^{\circ}$ 1. Rentabilidad Real Anual del Fondo de Pensiones (\%)

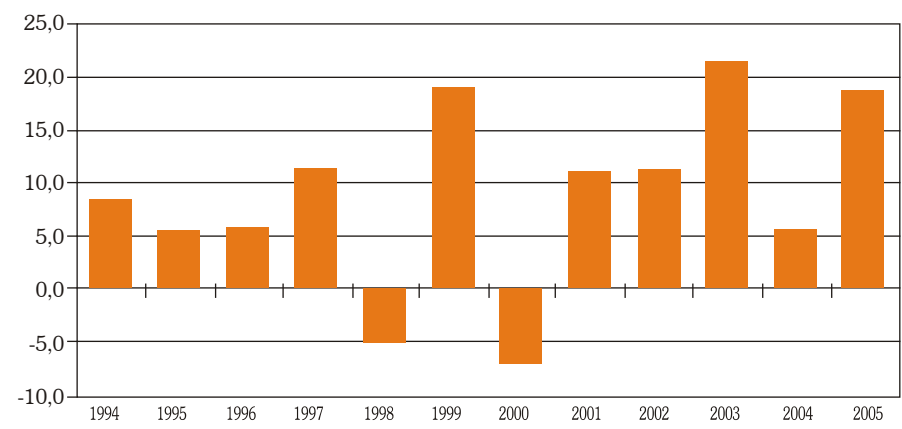

Fuente: SBS y AFP, boletines mensuales. 


\section{David Juan Sanchez Cruz}

Cuadro N. ${ }^{2}$ : Evolución Histórica de Carteras Administradas por AFP (en miles de nuevos soles)

\begin{tabular}{lllllll} 
& Horizonte & Integra & Prima & Profuturo & $\begin{array}{c}\text { Unión } \\
\text { Vida }\end{array}$ & $\begin{array}{c}\text { SPP } \\
\mathbf{1 0 0 \%}\end{array}$ \\
\hline \multirow{2}{*}{$\mathbf{2 0 0 0}$} & 2440262 & 3065612 & & 1431588 & 2762401 & 9699863 \\
& $25.2 \%$ & $31.6 \%$ & & $14.8 \%$ & $28.5 \%$ & \\
$\mathbf{2 0 0 1}$ & 3136276 & 3968857 & & 1885434 & 3476625 & 12467192 \\
& $25.2 \%$ & $31.8 \%$ & & $15.5 \%$ & $27.9 \%$ & \\
$\mathbf{2 0 0 2}$ & 4052485 & 5084052 & & 2440487 & 4329461 & 15906485 \\
& $25.5 \%$ & $32.0 \%$ & & $15.3 \%$ & $27.2 \%$ & \\
$\mathbf{2 0 0 3}$ & 5602060 & 7001917 & & 3442039 & 6009307 & 22055322 \\
& $25.4 \%$ & $31.7 \%$ & & $15.6 \%$ & $27.2 \%$ & \\
$\mathbf{2 0 0 4}$ & 6607139 & 8121509 & & 4116563 & 7062414 & 25907625 \\
& $25.5 \%$ & $31.3 \%$ & & $15.9 \%$ & $27.3 \%$ & \\
$\mathbf{2 0 0 5}$ & 8214313 & 10263382 & 875479 & 5139934 & 8080949 & 32574057 \\
& $25.2 \%$ & $31.5 \%$ & $2.7 \%$ & $15.8 \%$ & $24.8 \%$ & \\
\hline
\end{tabular}

Fuente: Elaboración del investigador, basado en información obtenida de la SBS.

Cuadro N. ${ }^{\circ}$ 3: Cartera Administrada del SPP por Instrumento Financiero: Evolución 2000 2005 (en miles de nuevos soles)

\begin{tabular}{|c|c|c|c|c|c|c|}
\hline Años & 2000 & 2001 & 2002 & 2003 & 2004 & 2005 \\
\hline I. INVERSIONES LOCALES & 9.049 .614 & 11.820 .808 & $\begin{array}{r}14755 \\
040\end{array}$ & $\begin{array}{r}20084 \\
404\end{array}$ & $\begin{array}{r}23306 \\
392\end{array}$ & $\begin{array}{r}29294 \\
688\end{array}$ \\
\hline 1. Gobierno & 874.872 & 1.716 .588 & $\begin{array}{r}2060 \\
487\end{array}$ & $\begin{array}{r}4294 \\
731\end{array}$ & $\begin{array}{r}6282 \\
443\end{array}$ & $\begin{array}{r}6602 \\
037\end{array}$ \\
\hline Certificados del BCRP & 264.436 & $\begin{array}{l}395 \\
767\end{array}$ & $\begin{array}{l}425 \\
711\end{array}$ & $\begin{array}{l}929 \\
644\end{array}$ & $\begin{array}{r}2694 \\
542\end{array}$ & $\begin{array}{r}1533 \\
365\end{array}$ \\
\hline Bonos del Gobierno Central & 98.763 & $\begin{array}{l}781 \\
282\end{array}$ & $\begin{array}{r}1372 \\
988\end{array}$ & $\begin{array}{r}2841 \\
633\end{array}$ & $\begin{array}{r}3093 \\
839\end{array}$ & $\begin{array}{r}4737 \\
312\end{array}$ \\
\hline Bonos Brady & 511.673 & $\begin{array}{l}539 \\
539\end{array}$ & $\begin{array}{l}261 \\
788\end{array}$ & $\begin{array}{l}523 \\
454\end{array}$ & $\begin{array}{l}494 \\
063\end{array}$ & $\begin{array}{l}331 \\
360\end{array}$ \\
\hline 2. Sistema Financiero & 3.299 .637 & 4.640 .317 & $\begin{array}{r}6410 \\
130\end{array}$ & $\begin{array}{r}6625 \\
809\end{array}$ & $\begin{array}{r}5162 \\
356\end{array}$ & $\begin{array}{r}6366 \\
347\end{array}$ \\
\hline $\begin{array}{l}\text { Certificados y Depósitos a } \\
\text { Plazo } \\
\text { Tít. Deuda Emitido Org. } \\
\text { Internacional Local }\end{array}$ & 1.463 .755 & 2.833 .895 & $\begin{array}{r}4040 \\
177 \\
672 \\
113\end{array}$ & $\begin{array}{r}3628 \\
683 \\
462 \\
826\end{array}$ & $\begin{array}{r}2006 \\
392 \\
21690\end{array}$ & $\begin{array}{r}2408 \\
266 \\
220 \\
423\end{array}$ \\
\hline $\begin{array}{l}\text { Bonos de Arrendamiento } \\
\text { Financiero }\end{array}$ & 1.228 .738 & $\begin{array}{r}1137 \\
383\end{array}$ & $\begin{array}{l}288 \\
731\end{array}$ & $\begin{array}{l}328 \\
707\end{array}$ & $\begin{array}{l}299 \\
794\end{array}$ & $\begin{array}{l}268 \\
628\end{array}$ \\
\hline Bonos Subordinados & 416.248 & $\begin{array}{l}383 \\
141\end{array}$ & $\begin{array}{l}264 \\
477\end{array}$ & $\begin{array}{l}283 \\
025\end{array}$ & $\begin{array}{l}316 \\
354\end{array}$ & $\begin{array}{l}324 \\
876\end{array}$ \\
\hline Otros Bonos Sector Financiero & 182.990 & $\begin{array}{l}269 \\
829\end{array}$ & 3039 & 2108 & $\begin{array}{l}293 \\
217\end{array}$ & $\begin{array}{l}352 \\
892\end{array}$ \\
\hline
\end{tabular}




\section{Portafolio de inversiones y desempeño del sistema privado de pensiones peruano: 2000 - 2005}

\begin{tabular}{|c|c|c|c|c|c|c|}
\hline Letras Hipotecarias & 7.906 & 4296 & 12083 & 12926 & 1254 & 797 \\
\hline Bonos Hipotecarios & & 11773 & $\begin{array}{r}1129 \\
509\end{array}$ & $\begin{array}{r}1907 \\
535\end{array}$ & 21059 & 26784 \\
\hline $\begin{array}{l}\text { Acciones y Valores } \\
\text { representativos sobre Acciones }\end{array}$ & & & & & $\begin{array}{r}2202 \\
595\end{array}$ & $\begin{array}{r}2763 \\
680\end{array}$ \\
\hline 3. Empresas no Financieras & 4.613 .795 & 5.125 .786 & $\begin{array}{r}5911 \\
517\end{array}$ & $\begin{array}{r}8599 \\
337\end{array}$ & $\begin{array}{r}10564 \\
323\end{array}$ & $\begin{array}{r}12578 \\
868\end{array}$ \\
\hline Papeles Comerciales & 104.542 & $\begin{array}{l}250 \\
252\end{array}$ & 261 & $\begin{array}{l}331 \\
326\end{array}$ & 73170 & $\begin{array}{l}000 \\
107 \\
685\end{array}$ \\
\hline Pagarés No Avalados LP & & & $\begin{array}{r}1727 \\
103\end{array}$ & $\begin{array}{r}2397 \\
054\end{array}$ & $\begin{array}{r}2335 \\
949\end{array}$ & 84821 \\
\hline $\begin{array}{l}\text { Bonos de Empresas no } \\
\text { Financieras }\end{array}$ & 1.701 .033 & $\begin{array}{r}1710 \\
000\end{array}$ & 90885 & $\begin{array}{l}144 \\
833\end{array}$ & $\begin{array}{l}581 \\
327\end{array}$ & $\begin{array}{r}2514 \\
178\end{array}$ \\
\hline Bonos para Nuevos Proyectos & & 72808 & $\begin{array}{r}3832 \\
314\end{array}$ & $\begin{array}{r}5926 \\
124\end{array}$ & $\begin{array}{r}7173 \\
851\end{array}$ & $\begin{array}{l}763 \\
754\end{array}$ \\
\hline $\begin{array}{l}\text { Acciones y Valores } \\
\text { representativos sobre Acciones }\end{array}$ & 2.808 .220 & 3.092 .727 & & & $\begin{array}{l}400 \\
026\end{array}$ & $\begin{array}{r}9108 \\
430\end{array}$ \\
\hline 4. Fondos de Inversión & 65.100 & 62.731 & $\begin{array}{l}123 \\
662\end{array}$ & $\begin{array}{l}228 \\
059\end{array}$ & $\begin{array}{l}370 \\
875\end{array}$ & $\begin{array}{l}906 \\
233\end{array}$ \\
\hline Cuotas de Fondos de Inversión & 58.349 & 55651 & $\begin{array}{l}116 \\
506\end{array}$ & $\begin{array}{l}228 \\
059\end{array}$ & $\begin{array}{l}370 \\
875\end{array}$ & $\begin{array}{l}906 \\
233\end{array}$ \\
\hline Bonos de Fondos de Inversión & 6.751 & 7081 & 7155 & - & - & - \\
\hline 5. Sociedades Titulizadoras & 196.210 & 275.385 & $\begin{array}{l}249 \\
245\end{array}$ & $\begin{array}{l}336 \\
468\end{array}$ & $\begin{array}{l}926 \\
394\end{array}$ & $\begin{array}{r}2841 \\
203\end{array}$ \\
\hline Bonos de Titulización & 196.210 & $\begin{array}{l}275 \\
385\end{array}$ & $\begin{array}{l}249 \\
245\end{array}$ & $\begin{array}{l}336 \\
468\end{array}$ & $\begin{array}{l}601 \\
183\end{array}$ & $\begin{array}{r}1690 \\
035\end{array}$ \\
\hline $\begin{array}{l}\text { Títulos con Derecho de } \\
\text { Participación }\end{array}$ & & & & & $\begin{array}{l}325 \\
212\end{array}$ & $\begin{array}{r}1151 \\
168\end{array}$ \\
\hline $\begin{array}{l}\text { II. INVERSIONES EN EL } \\
\text { EXTERIOR }\end{array}$ & 647.412 & 596.118 & $\begin{array}{r}1145 \\
207\end{array}$ & $\begin{array}{r}1936 \\
140\end{array}$ & $\begin{array}{r}2634 \\
420\end{array}$ & $\begin{array}{r}3300 \\
756\end{array}$ \\
\hline 1. Gobierno & & 389.303 & $\begin{array}{l}440 \\
276\end{array}$ & 255 & 165 & $\begin{array}{l}110 \\
163\end{array}$ \\
\hline Títulos de Deuda & & $\begin{array}{l}389 \\
303\end{array}$ & $\begin{array}{l}440 \\
276\end{array}$ & $\begin{array}{l}255 \\
979\end{array}$ & $\begin{array}{l}165 \\
271\end{array}$ & $\begin{array}{l}110 \\
163\end{array}$ \\
\hline 2. Sistema Financiero & & & 25075 & 37583 & $\begin{array}{l}138 \\
419\end{array}$ & 91014 \\
\hline Bonos de Agencias & & & 20594 & 7522 & - & - \\
\hline $\begin{array}{l}\text { Certificados y Depósitos a } \\
\text { Plazo }\end{array}$ & & & 4481 & 30061 & $\begin{array}{l}138 \\
419\end{array}$ & 91014 \\
\hline 3. Empresas no Financieras & 647.412 & 30.400 & 6444 & 8075 & 18311 & 8260 \\
\hline $\begin{array}{l}\text { Acciones y Valores } \\
\text { representativos sobre Acciones }\end{array}$ & 647.412 & 30400 & 6444 & 8075 & 18311 & 8260 \\
\hline 4. Administradoras de Fondos & & 176.416 & $\begin{array}{l}673 \\
412\end{array}$ & $\begin{array}{r}1634 \\
503\end{array}$ & $\begin{array}{r}2312 \\
419\end{array}$ & $\begin{array}{r}3091 \\
320\end{array}$ \\
\hline Fondos Mutuos & & $\begin{array}{l}176 \\
416\end{array}$ & $\begin{array}{l}673 \\
412\end{array}$ & $\begin{array}{r}1634 \\
503\end{array}$ & $\begin{array}{r}2312 \\
419\end{array}$ & $\begin{array}{r}3091 \\
320\end{array}$ \\
\hline III. OPERACIONES EN TRÁNSITO & 2.838 & 50265 & 6238 & 34778 & (33 186) & (21 387) \\
\hline TOTAL & 9.699 .863 & 12.467 .191 & $\begin{array}{r}15906 \\
485\end{array}$ & $\begin{array}{r}22055 \\
322\end{array}$ & $\begin{array}{r}25907 \\
625\end{array}$ & $\begin{array}{r}32574 \\
057\end{array}$ \\
\hline Fondo de Pensiones & 9.598 .637 & 12350 & $\begin{array}{r}15753 \\
704\end{array}$ & $\begin{array}{r}21843 \\
896\end{array}$ & $\begin{array}{r}25650 \\
770\end{array}$ & $\begin{array}{r}32222 \\
592\end{array}$ \\
\hline Encaje Legal & 101.226 & $\begin{array}{l}116 \\
881\end{array}$ & $\begin{array}{l}152 \\
782\end{array}$ & $\begin{array}{l}211 \\
426\end{array}$ & $\begin{array}{l}256 \\
855\end{array}$ & $\begin{array}{l}351 \\
466\end{array}$ \\
\hline
\end{tabular}

Fuente: Elaboración del investigador, basado en boletines mensuales SBS. 


\section{David Juan Sanchez Cruz}

Los instrumentos de inversión en que pueden incurrir las AFP son regulados por el DS² 054-97-EF-TUO-Ley SPP:

El BCRP establece los límites de inversión operativos. La actual estructura de límites de inversión por instrumentos vigentes fue establecida el 2 de junio de 2003, por Circular BCRP N. ${ }^{\circ}$ 016-03-EF/90. Sobre ello, se señala que la regulación fomenta la adquisición de valores de bajo riesgo relativo.

La diversificación por sectores económicos ha permitido reducir el riesgo del fondo. A diciembre 2005 la cartera administrada se encuentra distribuida en, por lo menos, diez sectores económicos distintos, siendo los principales el gobierno nacional, minería, intermediación financiera y energía.

\section{MARCO TEÓRICO}

\subsection{Eficiencia de Mercado}

En términos de inversionistas, se llama un mercado eficiente cuando la información está ampliamente disponible y en forma barata para los inversores, y toda la información relevante y alcanzable está ya reflejada en los precios de los títulos; razón por la cual las compras o ventas en un mercado eficiente no pueden ser transacciones de VAN positivo. Por consiguiente, un inversor no le puede ganar sistemáticamente al mercado (entendiéndose por mercado el índice de referencia o benchmark del portafolio).

La teoría de los mercados eficientes está basada en la Teoría del Camino Azaroso (random-walk theory). Un camino azaroso es aquel en que los futuros pasos o direcciones no pueden preverse sobre la base de las acciones pasadas. Cuando el término es aplicado a los mercados de capitales, significa que los movimientos de los activos no pueden ser predichos. Un defensor de la eficiencia de mercado, ante la pregunta: ¿Cuál es el mejor predictor de los precios futuros de un activo?, contestaría: "El precio de hoy".

2 Decreto Supremo N. ${ }^{\circ}$ 054-97-EF, Texto único Ordenado de la Ley del Sistema Privado de Administración de Fondos de Pensiones. 


\section{Portafolio de inversiones y desempeño del sistema privado de pensiones peruano: 2000 - 2005}

Gráfico N. ${ }^{\circ}$ 5. Precio de la acción al detectarse un ciclo.

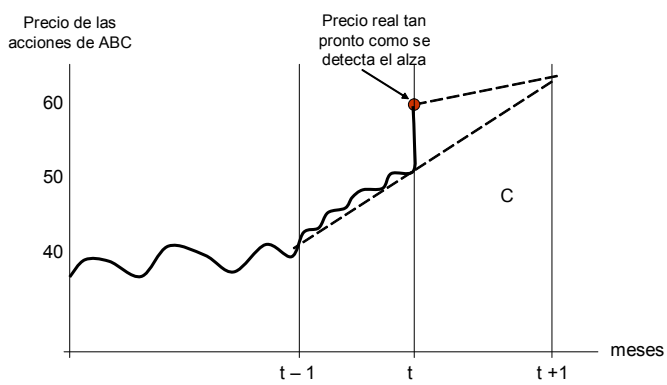

Fuente: Richard A. Brealey, Stewart C. Myers.

Esto también se explica pues la eficiencia está relacionada directamente con la disponibilidad de la información para los inversores; dado que la información se ve reflejada en los precios de los activos, cuánto mejor y más amplia sea esta información, mejor va a estar reflejado en el precio de estos activos su real y justo valor.

Harry Roberts, en su documento "Statistical versus clinical prediction of the stock market" (1967), define tres niveles de eficiencia:

1. Forma Débil de Eficiencia. Cuando los precios reflejan toda la información contenida en la evolución de los precios pasados; y contando con toda la información pasada disponible, no se le puede ganar al mercado en forma sistemática. O sea, el estudio de los precios pasados no son de utilidad para predecir precios futuros.

2. Forma Semifuerte de Eficiencia. Cuando los precios reflejan no solo la evolución de los precios pasados, sino además toda la información publicada disponible. O sea, el análisis de los balances de la empresa, ir a conocer la fábrica, conocer los planes de expansión, entre otros, no sirven para predecir los precios futuros y ganarle sistemáticamente al mercado. El análisis fundamental es una técnica de inversión que basa sus predicciones en el estudio de la información de la empresa, la industria y la economía. Si el mercado resultara eficiente desde el punto de vista semi-fuerte, el análisis fundamental no tendría razón de existir. 


\section{David Juan Sanchez Cruz}

3. Forma Fuerte de Eficiencia. Cuando los precios reflejan no solo la información pública, sino toda la información que se puede adquirir mediante concienzudos análisis de la empresa y la economía. En tal mercado no encontraríamos ningún gestor de inversiones que le pueda ganar sistemáticamente al mercado.

\subsection{Teoría del Portafolio}

Definida como el análisis cuantitativo de la administración óptima del riesgo, para un conjunto de activos financieros.

En 1952, Harry M. Markowitz publicó una investigación que dio origen a la Teoría de Portafolio ${ }^{3}$ moderna. El principal aporte de Markowitz fue el de modelar la racionalidad del inversionista en el mercado de capitales. Esta teoría muestra la relación que debe de existir entre el riesgo y el retorno de un activo cualquiera; para ello, se maximiza el rendimiento esperado a un cierto nivel de riesgo, o se minimiza el riesgo a un nivel esperado de rendimiento.

\subsubsection{Supuestos del Modelo de la Teoría de Portafolio}

Existen distintos supuestos sobre los cuales se basa esta teoría, entre ellos tenemos:

a. Como inversionista, uno busca maximizar el retorno de sus inversiones a un nivel dado de riesgo.

b. Los inversionistas estiman el riesgo del portafolio basándose en la variabilidad del retorno esperado.

c. Los inversionistas basan sus decisiones únicamente en el riesgo y el retorno esperado, para que así sus curvas de utilidad estén en función del retorno esperado y de la variancia esperada.

d. Para un nivel dado de riesgo, los inversionistas prefieren un alto retorno, y ante un nivel de retorno dado, los inversionistas prefieren un menor riesgo.

3 Markowitz, Harry M. "Portfolio Selection". En: Journal of Finance, 1952. Derivó la tasa de retorno esperada de un portafolio y calculó el riesgo esperado. 
Portafolio de inversiones y desempeño del sistema privado de pensiones peruano: 2000 - 2005

\subsubsection{Medidas del Riesgo}

La volatilidad 4 es una medida utilizada comúnmente para medir el grado de riesgo de un activo como las acciones. La volatilidad está relacionada con el rango de posibles tasas de rendimiento derivadas de poseer las acciones y la probabilidad de que ocurren. La volatilidad de una acción será mayor cuanto más amplio sea el rango de posibles resultados o sucesos y mayores sean las probabilidades de que esos rendimientos se ubiquen en los extremos del rango.

La tasa esperada de rendimiento (la media), llamada también Rendimiento Esperado, se define como la suma de todos los resultados posibles de cada tasa de rendimiento posible, multiplicado por la probabilidad respectiva de que ocurran:

$$
\begin{gathered}
E(R)=w_{1} R_{1}+w_{2} R_{2}+\ldots . .+w_{n} R_{n} \\
E(R)=\sum_{i=1}^{n} w_{i} R_{i}
\end{gathered}
$$

La estadística que se usa con mayor frecuencia en finanzas para cuantificar y medir la volatilidad de la distribución de probabilidades del rendimiento de acciones es la desviación estándar, y se calcula de la siguiente manera:

$$
\sigma=\sqrt{\sum_{i=1}^{n} P_{i}\left(r_{i}-E(r)\right)^{2}}
$$

Cuanto mayor sea la desviación estándar, mayor será la volatilidad de las acciones.

\subsection{Modelo CAPM (Capital Asset Pricing Model)}

Desde su aparición, de la mano de William Sharpe, en 1964, el CAPM (en español: Modelo de Valuación de Activos de Capital) se ha transformado en uno de los modelos más utilizados en el ámbito financiero.

Un primer aporte importante del CAPM en la investigación económica financiera es agrupar los riesgos en:

- El Riesgo Sistemático (o de mercado), que muestra la sensibilidad de la rentabilidad de la empresa ante el conjunto de la economía que amenaza la totalidad

4 El costo de asegurarse contra un riesgo depende directamente de la volatilidad. 


\section{David Juan Sanchez Cruz}

del mercado. Este riesgo no puede ser eliminado mediante diversificación, y por tanto este será el único componente de riesgo que será premiado por el mercado de capitales. Bajo el supuesto de que los directivos empresariales buscan maximizar la riqueza del accionista, el CAPM implica que los directivos deberían gestionar el riesgo sistemático, ya que este riesgo es no diversificable, y en consecuencia, resulta relevante para los inversores.

- El Riesgo No Sistemático (único o propio). Es consecuencia del hecho que muchos de los peligros que rodean a una determinada empresa son específicos suyos; este riesgo puede ser potencialmente eliminado de la cartera mediante diversificación de las inversiones. Por tanto, este riesgo debería resultar irrelevante para el directivo de la empresa, ya que resulta mucho más fácil y menos costosa la diversificación de la cartera de inversiones del accionista, que diversificar los negocios de la empresa con el objetivo de reducirlo.

El CAPM se sustenta en supuestos sobre el mercado de capitales, destacando que este mercado es perfecto y está en equilibrio; todos los inversionistas determinan el portafolio óptimo empleando el enfoque de Markowitz. Es importante además, tener en cuenta los supuestos siguientes:

1. Los inversionistas evalúan los portafolios tomando en cuenta los retornos esperados y la desviación estándar de los diversos portafolios en un horizonte de un período.

2. Existe la no saciedad entre los inversionistas. Esto implica que dados dos portafolios idénticos, escogerán aquel de mayor retorno esperado.

3. Los inversionistas son adversos al riesgo. Dados dos portafolios iguales, se escogerá aquel de menor desviación estándar.

4. Los valores son infinitamente divisibles. Si un inversionista lo desea, puede adquirir la fracción de una acción.

5. Existe una tasa libre de riesgo a la cual el inversionista puede invertir o pedir préstamos.

6. Los impuestos y los costos de transacción son irrelevantes.

7. Todos los inversionistas tienen el mismo horizonte de un período.

8. La tasa libre de riesgo es la misma para todos los inversionistas. 


\section{Portafolio de inversiones y desempeño del sistema privado de pensiones peruano: 2000 - 2005}

9. Existe información perfecta.

10.Los inversionistas tienen expectativas homogéneas.

A pesar que los supuestos del modelo no necesariamente se cumplen en la vida real, la capacidad predictiva del modelo ha demostrado ser efectiva.

La Teoría CAPM es una herramienta comúnmente usada para medir el riesgo de los activos. Esta teoría se basa en la premisa de que los retornos de un activo o portafolio dependen solamente del riesgo sistemático o de mercado. El modelo plantea una relación lineal entre los retornos y el riesgo.

Donde:

$$
E\left(R_{j}\right)=R_{f}+\beta_{j}\left\lfloor E\left(R_{M}\right)-R_{f}\right\rfloor
$$

$E\left(R_{j}\right)$ : Tasa de retorno esperada sobre un activo o portafolio particular. $R_{f}$ : Tasa libre de riesgo.

$\beta_{\mathrm{j}}$ : $\quad$ Riesgo asumido por el activo o "portafolio $\mathrm{j}$ ", y se define como la covarianza entre los retornos del activo riesgoso " $\mathrm{j}$ " y los del portafolio del mercado "m", dividido por la varianza del portafolio del mercado.

$\mathrm{E}\left(\mathrm{R}_{\mathrm{M}}\right)$ : Retorno del portafolio de mercado esperado.

El CAPM implica que a la mayoría de los inversionistas les daría el mismo resultado combinar de manera pasiva el activo sin riesgo con un fondo índice que mantuviera activos riesgosos en las mismas proporciones que en la cartera de mercado, o bien buscar valores en forma activa y tratar de "aventajar" al mercado. Los inversionistas especialmente diligentes y competentes obtienen excelentes recompensas por sus esfuerzos, pero con el tiempo la competencia entre ellos reduce esas recompensas al mínimo necesario para inducirlos a desempeñar su trabajo. El resto de nosotros podemos beneficiarnos de su trabajo invirtiendo de manera pasiva.

\subsection{Beta y primas de riesgo}

Los precios de equilibrio de activos y los rendimientos esperados son tales que los inversionistas conocedores mantienen de buena gana los activos que tienen en sus carteras óptimas. Con la idea que a los inversionistas se les debe compensar, en términos del rendimiento esperado por asumir riesgos, definimos el riesgo de un valor por la 


\section{David Juan Sanchez Cruz}

magnitud de su rendimiento esperado de equilibrio. Por consiguiente, el riesgo del valor $A$ es mayor que el riesgo del valor $B$ si en equilibrio el rendimiento esperado de $A$ excede el rendimiento esperado de B. Si revisamos entre las carteras óptimas (eficientes), cuánto más grande sea la desviación estándar de su rendimiento, más grande será el rendimiento de equilibrio $E(r)$, y por ende, más grande será el riesgo. Por lo tanto, el riesgo de una cartera eficiente se mide por $\sigma$. Sin embargo, la desviación estándar del rendimiento no mide, por lo general, el riesgo de valores en el CAPM. En lugar de eso, la medida general del riesgo de un valor es la beta (la letra griega $\beta$ ). Técnicamente:

$$
\beta \equiv \frac{\sigma_{j M}}{\sigma_{M}^{2}}
$$

Donde:

$\sigma_{\mathrm{j}}$ : Expresa la covarianza entre el rendimiento del valor $\mathrm{j}$ y el rendimiento de la cartera de mercado.

$\sigma_{\mathrm{M}}^{2}$ : Expresa la varianza del rendimiento de la cartera de mercado.

De acuerdo con el CAPM, en equilibrio, la prima de riesgo de cualquier activo es igual a su beta multiplicada por la prima de riesgo de la cartera de mercado. Esta relación se expresa:

$$
E\left(R_{j}\right)-R_{f}=\beta_{j}\left\lfloor E\left(R_{M}\right)-R_{f}\right\rfloor
$$

Donde:

$E\left(R_{j}\right)-R_{f}$ : Prima por riesgo individual

$E\left(R_{M}\right)-R_{f}$ : Prima por riesgo de mercado

\section{EL RIESGO DE MERCADO Y LA BETA}

Para conocer la contribución de un título individual al riesgo de una cartera bien diversificada, se necesita medir su riesgo de mercado, lo que equivale a medir su sensibilidad respecto a los movimientos del mercado, a la que se denomina beta $\beta$.

Beta se puede interpretar como el grado de respuesta de la variabilidad de los rendimientos de la acción a la variabilidad de los rendimientos del mercado. Si $\beta_{i}>1$, entonces tenemos que las variaciones en los rendimientos del valor $i$ serán mayores a las variaciones del rendimiento del mercado. Por lo contrario, si $\beta_{i}<1$, entonces el valor $i$ 


\section{Portafolio de inversiones y desempeño del sistema privado de pensiones peruano: 2000 - 2005}

será menos riesgoso que el rendimiento del mercado. Si $\beta_{\mathrm{i}}=1$; el rendimiento del valor i variará en la misma proporción que la variación del rendimiento de mercado. El mercado es la cartera de todas las acciones, por tanto la "acción media" tiene una beta de 1 .

Si construimos una cartera que contenga un gran numero de acciones (unas 500, elegidas aleatoriamente de todo el mercado), estaríamos consiguiendo el mercado en sí, o una cartera muy próxima a él. Beta sería 1 y la correlación con el mercado sería 1.

\subsection{Diversificación Eficiente}

Diversificar es dividir una inversión en muchos activos riesgosos en vez de concentrarla en uno solo. El principio de diversificación dice que mediante la diversificación de activos riesgosos, la gente a veces puede lograr una reducción de su exposición global al riesgo, sin reducción alguna de su rendimiento esperado.

La diversificación se produce porque los precios de las diferentes acciones no evolucionan en idéntico sentido. Las estadísticas hacen referencia a esto cuando indican que los cambios en los precios de las acciones están imperfectamente correlacionados. Una caída en el valor de una de las acciones es compensada por la subida en el precio de otra.

En el Gráfico N. ${ }^{\circ} 6$ apreciamos que mediante la diversificación es posible reducir el riesgo no sistemático.

Gráfico N. ${ }^{\circ}$ 6. Riesgo de Portafolio.

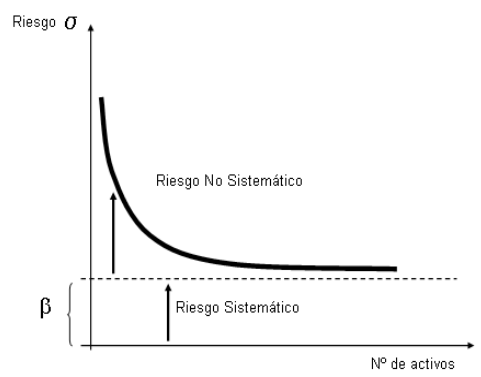

Fuente: Richard A. Brealey, Stewart C. Myers. 


\section{David Juan Sanchez Cruz}

El riesgo propio o no sistemático puede ser eliminado por la diversificación, pero el riesgo de mercado o sistemático no se puede evitar.

\subsubsection{El conjunto factible y el conjunto eficiente}

El conjunto factible consiste en todos los posibles portafolios que pueden conformarse a partir de "n" títulos riesgosos. Este se representa gráficamente en el plano retorno esperado - desviación estándar. En el Gráfico N. ${ }^{\circ} 7$ se muestra la forma que tendría el conjunto factible. En general, la gráfica del conjunto tendrá una forma similar a la de un "paraguas" 5 .

Gráfico N. ${ }^{\circ}$ 7. Conjunto Factible.

Fuente: Stephen Ross. Finanzas Corporativas. Conjunto Factible

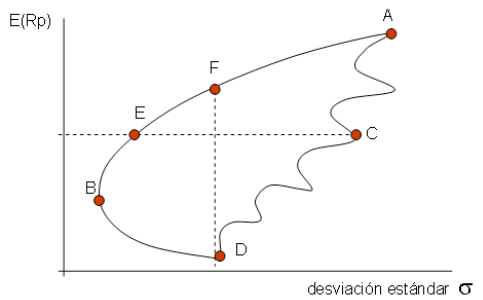

Sin embargo, un inversionista no necesariamente escogerá cualquier portafolio del conjunto factible. Algunos portafolios del conjunto serán mejores que otros. Así, un agente racional limitará sus posibilidades de elección a aquellos portafolios que sean los más eficientes del conjunto factible.

Asumiendo que el inversionista es adverso al riesgo, siempre escogerá una combinación de títulos riesgosos tales que:

a. Dado un nivel de riesgo determinado, ofrezca la rentabilidad máxima.

b. Dado un nivel de rentabilidad determinado, ofrezca un riesgo mínimo.

Tomando en cuenta ambos criterios, se determina un conjunto eficiente de portafolios que se encuentra delimitado por el segmento $A B$ del Gráfico N. ${ }^{\circ} 7$. El punto $D$ del set factible no puede formar parte del conjunto eficiente, ya que para ese nivel de riesgo se puede adquirir un portafolio de mayor rentabilidad (F). Asimismo, el punto $C$ tam-

5 Matemáticamente, el área bajo la curva debe ser un conjunto cóncavo en el sentido estricto. 


\section{Portafolio de inversiones y desempeño del sistema privado de pensiones peruano: 2000 - 2005}

poco puede estar dentro del set eficiente; para dicha rentabilidad, puede conformarse un portafolio de menor riesgo (E). Ningún inversionista querrá tener portafolios con un rendimiento esperado menor al portafolio de varianza mínima que viene a ser el punto B. Aunque toda la curva $D A$ se llama conjunto factible, los inversionistas solo consideran la curva $B A$, llamada conjunto eficiente o frontera eficiente ${ }^{6}$.

Una propiedad que siempre debe cumplirse en el set eficiente es la concavidad. Esto es, la frontera eficiente que surge de unir los puntos BFEA del Gráfico N ${ }^{\circ} 7$.

\subsection{El Conjunto Eficiente en presencia de Títulos Libres de Riesgo}

Cuando existe la posibilidad de comprar títulos libres de riesgo, o pedir préstamos a tasas libres de riesgo, el set eficiente cambia de forma. Si un inversionista hubiera escogido el portafolio $A$ del Gráfico N. ${ }^{\circ} 8$, y existiera un título libre de riesgo con un retorno igual a $\mathrm{R}_{\mathrm{f}}$, entonces la recta $R$ - $A$ indicaría todas las combinaciones posibles que podrían formarse entre el título libre de riesgo y el portafolio de títulos riesgosos. Sin embargo, estas combinaciones no son las óptimas. Si en lugar del portafolio $A$ se escogiera el portafolio $B$, entonces las combinaciones de $R$ - B superarían a las de $R-A$, debido a que se podría obtener una mayor rentabilidad para cada nivel de riesgo. Es posible determinar infinidad de portafolios del set eficiente que podrían entrar en combinación con el título libre de riesgo, pero solamente existe un portafolio óptimo. En el Gráfico N. ${ }^{\circ} 8$, se puede apreciar que el portafolio óptimo (punto $\mathrm{T}$ ) es aquel que maximiza la pendiente de la recta que une el punto asociado al título libre de riesgo y el set eficiente inicial.

Gráfico N. ${ }^{\circ}$ 8. Frontera Eficiente en presencia de títulos libres de riesgo.

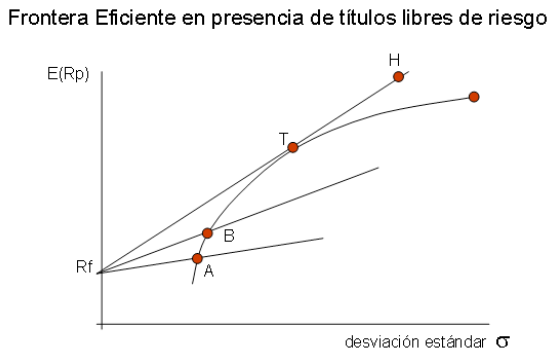

Fuente: Maria B. Collatti. Teoría de Carteras.

6 Stephen A., Ross; Randolph W., Westerfield; Jeffrey Jaffe. Finanzas Corporativas. 


\section{David Juan Sanchez Cruz}

\subsection{Administración de Portafolio}

Los inversionistas, quienes proveen los fondos necesarios a los administradores de portafolio o cartera, deben continuamente tomar decisiones, y estas pueden ser intuitivas o bajo el criterio de rendimiento-riesgo.

Una organización de administración de inversiones reúne economistas, técnicos y otros expertos del mercado, quienes hacen proyecciones acerca de la economía y los mercados financieros, lo que se comunica a los analistas de valores. Los analistas hacen predicciones acerca de los valores de los que son responsables y se resumen en clasificaciones, información que se trasmite al comité de inversiones.

El administrador de portafolio o cartera es el responsable de administrar los activos financieros, y puede: 1) ser activo o pasivo; 2) usar procedimientos explícitos o implícitos. La tendencia es hacia operaciones controladas.

\subsubsection{Selección de portafolios de Inversión}

Se deben hacer las siguientes precisiones: en la actualidad los inversores pueden ser individuos o instituciones (de allí la denominación de inversores institucionales); el mismo inversor puede determinar el destino de sus inversiones o hacerlo a través de un tercero que se denominará portafolio manager o administrador de carteras.

Ahora bien, ¿en qué se invierte? La macroeconomía enseña que los bienes producidos en un determinado período de tiempo por la sociedad o se consumen o se invierten. Es decir, podemos invertir tanto capitales, como activos; a su vez, se invierten en otro conjunto de capitales o activos, que se agrupan dentro de una cartera de inversiones, la cual puede ser a su vez una multitud de carteras.

El objetivo central de la administración de carteras es ciertamente la construcción de un portafolio. Para lograr esto, un portafolio manager o administrador de inversiones debe tener en cuenta los siguientes aspectos:

1. Definir qué clase de activos van a ser incluidos en el portafolio

2. Perfil de riesgo del inversor 


\section{Portafolio de inversiones y desempeño del sistema privado de pensiones peruano: 2000 - 2005}

3. Principios de diversificación

4. Longitud del plazo de inversión, y si es uno o varios períodos

5. Definición del índice de referencia o "benchmark" del portafolio

6. Asignación estratégica de cada clase de activo en el largo plazo

7. Asignación táctica de cada clase de activos en el corto plazo

8. Estrategia de selección a usar dentro de cada clase de activo.

\subsection{Indicadores de Desempeño}

Los índices a trabajar tienen como "benchmark" la Línea de Mercado de Capitales (LMC) y la Línea de Mercado de Activos (LMA).

\subsubsection{Indicador de Sharpe}

William F. (Sharpe), en 1966, propone un cociente que viene determinado por la pendiente de la recta que conecta la cartera sin riesgo a la cartera riesgosa. Representa la prima de riesgo unitaria para el inversor. En otras palabras, proporciona el nivel de rendimiento por unidad de riesgo:

Donde

$$
\mathrm{S}_{\mathrm{p}}=(\mathrm{Rp}-\mathrm{Rf}) / \sigma_{\mathrm{p}}
$$

$\mathrm{S}_{\mathrm{p}}$ : Cociente de Sharpe de la cartera riesgosa $\mathrm{P}$

$\mathrm{Rp}$ : Rendimiento de la cartera riesgosa $\mathrm{P}$

$\mathrm{Rf}$ : Tasa sin riesgo

$\sigma_{\mathrm{p}}$ : Volatilidad (medida de riesgo) de la cartera riesgosa P.

Al utilizar como medida de riesgo la desviación estándar del portafolio; es decir, considera el riesgo total; por tanto implícitamente está evaluando la manera en que el administrador ha diversificado el portafolio.

\subsubsection{Indicador de Treynor}

El cociente de Treynor (Tp) propuesto en 1965 por Jack Treynor, viene determinado por la siguiente expresión: 


\section{David Juan Sanchez Cruz}

$$
T_{p}=\left(R_{p}-R_{f}\right) / \beta_{p}
$$

Donde:

Tp : Rendimiento del portafolio por unidad de riesgo

$\mathrm{Rf}$ : Rendimiento de un activo de libre de riesgo

Rp: Rendimiento del portafolio evaluado

$\beta_{\mathrm{p}}$ : Parámetro del modelo CAPM.

Este cociente representa la pendiente de la recta que conecta la cartera sin riesgo a la cartera riesgosa $P$ del inversor. Vemos que informa de la prima de riesgo $\left(R_{p}-R_{f}\right) a$ la medida de riesgo sistemática de la cartera.

Elegimos también el cociente más elevado posible. Para un mismo riesgo sistemático, elegimos pues la rentabilidad más elevada.

La medida de Treynor hace la hipótesis que los inversores pueden elegir entre varias carteras riesgosas (lo que no es el caso con el cociente de Sharpe).

\subsubsection{El Market Timing y la Selectividad}

El market timing es la habilidad del administrador de un fondo para anticiparse sistemáticamente a los movimientos del mercado, logrando mayores retornos o, en otro caso, menores pérdidas.

Si existe una estrategia de timing exitosa, el retorno del portafolio sería una función creciente y convexa del retorno del mercado. Supongamos que la bolsa de valores tuviera próximamente un período de auge. Si un administrador de portafolio pudiera prever esta situación, entonces compondría su portafolio de valores sumamente sensibles a los movimientos del mercado de capitales.

La habilidad de selectividad de un administrador es su capacidad para elegir aquellos activos que el mercado ha sub o sobre valuado por carecer de información suficiente o por tener información incorrecta. La utilización de información privilegiada sobre algunos activos en particular puede generar de hecho un mayor retorno que el de mercado. En general, un administrador que tiene la habilidad de selectividad, comprará 


\section{Portafolio de inversiones y desempeño del sistema privado de pensiones peruano: 2000 - 2005}

activos que en promedio tendrán un retorno mayor al estimado por el mercado; y por el contrario, venderá aquellos que él considere estén sobre valorados.

\subsubsection{Indicador de Jensen}

Esta medida propuesta por Michael C. Jensen, en 1968, particularmente se basa también en el riesgo sistemático y en el modelo de valuación de activos de capital (CAPM). A partir de estos resultados, Jensen determina la prima de riesgo, para un nivel de riesgo sistemático dado, a la cual puede pretender todo fondo de colocación que practica una política de buy and hold. Va del principio que si el gestor de cartera anticipó correctamente los precios de equilibrio del mercado y que se propone diversificar su cartera de manera eficiente en el sentido de Markowitz, la cartera debe entonces situarse sobre la LMA:

El índice de Jensen es precisamente el valor obtenido para alfa:

$$
\alpha=\left(R p-R_{F}\right)-\beta_{p}\left(R m-R_{F}\right)+e_{t}
$$

Donde:

$\alpha$ : Índice de Jensen ${ }^{7}$

$\mathrm{Rp}:$ Rendimiento del portafolio

$\mathrm{R}_{\mathrm{F}}$ : Rendimiento del activo libre de riesgo

$\mathrm{R}_{\mathrm{m}}$ : Rendimiento del mercado

$\beta$ : Sensibilidad del portafolio a las fluctuaciones en el mercado de valores

$e_{t}$ : Término de error que se comporta como ruido blanco.

\subsubsection{Indicador de Treynor-Mazuy}

En 1966, Treynor y Mazuy fueron los primeros en proponer una relación cuadrática para determinar una medida del market timing. Esta medida se emplea para evaluar la existencia de timing ${ }^{8}$, se entiende por ello la habilidad del administrador de

7 Término aleatorio que posee una distribución normal, con media cero y varianza constante.

8 Para ello cuentan con algunas técnicas: una consiste en ajustar el $\square$ promedio del portafolio, anticipando cambios en el mercado. Otra consiste en comparar gráficamente el retorno del fondo con el retorno de mercado. 


\section{David Juan Sanchez Cruz}

un fondo para anticiparse a la evolución del mercado. Para ello se estima la ecuación de Treynor-Mazuy, que es semejante a la ecuación de Jensen, pero se le añade un término cuadrático:

$$
\left(R_{p}-R_{F}\right)=\alpha+\beta_{1}\left(R_{m}-R_{F}\right)+\beta_{2}\left(R_{m}-R_{F}\right)^{2}+e_{t}
$$

Donde

$\mathrm{R}_{\mathrm{p}}, \mathrm{R}_{\mathrm{P}} \mathrm{R}_{\mathrm{m}}$ : Son los rendimientos de la cartera $\mathrm{P}$, de la tasa sin riesgo, y de la cartera de mercado $M$, respectivamente;

$\alpha$ Mide la selectividad; $\beta_{1}$ riesgo de mercado de portafolio (sensibilidad); $\beta_{2}$ mide el market timing del administrador; $e_{t}$ error residual con media cero. Según esta ecuación, si los $\beta_{2}$ son positivos y significativos, el administrador del fondo de inversión tiene una capacidad de timing. Según el estudio de los dos autores (Treynor y Mazuy) sobre 57 fondos de inversión, encontraron solo uno que posee esta aptitud.

\section{ANÁLISIS DE PORTAFOLIO}

En las secciones anteriores hemos visto los requerimientos para la prueba de eficiencia de mercado, la organización de portafolios y su evaluación, el modelo de valuación de activos de capital y desempeño financiero. A continuación, procedemos a desarrollar los modelos planteados.

\subsection{Búsqueda y tratamiento de la data}

La data obtenida corresponde al periodo enero 2000 - diciembre 2005, en periodos mensuales, y presenta ciertas dificultades, las más relevantes se tratan a continuación:

a) La tasa de interés libre de riesgo

Los mercados de capitales tienen como benchmark del activo libre de riesgo más generalizado, a los bonos del gobierno norteamericano (T- Bills). En el Perú, hasta fines de los 90, los investigadores empleaban la tasa de depósitos del Banco de Crédito 


\section{Portafolio de inversiones y desempeño del sistema privado de pensiones peruano: 2000 - 2005}

del Perú. Para el 2004, las instituciones rectoras del mercado financiero: BCRP, SBS, CONASEV, se esfuerzan por establecer una curva de rendimientos para el mercado nacional, la cual proporcione una tasa libre de riesgo. Para efectos prácticos, la autoridad monetaria toma como referencia los certificados de deposito del Banco Central de Reserva del Perú (CDBCRP) ${ }^{9}$, como "Proxy" de la tasa libre de riesgo, publicadas en la Nota Semanal del BCRP.

Siguiendo la metodología de la Teoría de Portafolio, en el presente trabajo, se asume la existencia de una única tasa a la cual se puede prestar y pedir prestado; es decir, supone que la tasa activa es igual a la tasa pasiva.

b) El portafolio de mercado

Se opta por la solución de utilizar un portafolio "proxy" del verdadero portafolio de mercado, aparece entonces el problema de la adecuada elección del portafolio "proxy".

Como proxy del portafolio de mercado se toma los índices selectivo y general de la Bolsa de Valores de Lima (mensuales), publicados en el resumen mensual de la BVL. Del trabajo realizado en el contexto de riesgo-retorno, se encontró que el ISBVL como más eficiente que el IGBVL, optando por descartar este último.

c) El portafolio riesgoso

Se obtuvo los retornos mensuales del SPP (para cada AFP) del boletín mensual de la Superintendencia de Banca y Seguros (SBS) y AFPs. La data completa fue llevada a términos constantes, corregidos por el Índice de Precios al Consumidor (IPC: abril $1998=100$ ) y anualizada. Toda esta información es posible apreciarla en el Anexo A.

\subsection{Modelación Financiera}

La modelación realizada al portafolio de activos financieros de las AFP, teniendo como proxy del activo libre de riesgo a la tasa del saldo de los CDBCRP, y proxy del portafolio de mercado al ISBVL, ha tenido el siguiente procedimiento:

9 Consultas realizadas por el investigador a funcionarios del BCRP y del MEF. 


\section{David Juan Sanchez Cruz}

Las Pruebas de Eficiencia de mercado para cada AFP, se usan para determinar si en realidad los mercados son perfectamente eficientes, razonablemente eficientes o no son eficientes en absoluto. Entre las diversas metodologías como el estudio de eventos, búsqueda de patrones en precios de valores, y examen de administradores de inversiones profesionales, se optó por el estudio de eventos, puesto que permite determinar qué tan rápido pueden reaccionar los precios de los activos ante la liberación de información: ¿Reaccionan rápido o lentamente? ¿Después de la fecha de anuncio, los rendimientos son anormalmente altos, bajos o normales? Del análisis, se desprende que la reacción de las cuatro AFP a las noticias es rápida y sostenida, evidencia que observamos en el tercer trimestre de 2005, por lo cual se puede afirmar que el mercado en que se desenvuelve el SPP es eficiente, por lo menos en su forma débil.

El análisis de inversión sustentado en la teoría de portafolio de Harry Markowitz, se inicia con el Modelo CAPM, el cual tiene como característica importante que en equilibrio, cada valor debe tener una proporción diferente de cero en la composición de la cartera de tangencia, que corresponde a las proporciones de la cartera de mercado. También vemos que los valores mostrados por los betas indicarían que las AFP, en la conformación de sus portafolios, son poco sensibles al índice de mercado representado por el ISBVL; esto también es posible comprobar en el análisis gráfico de reacción ante liberación de noticias, los que presentan baja volatilidad. Luego para el análisis de equilibrio se trabaja la formación de portafolios para las AFP, tomando como insumos el portafolio de activos de cada AFP y el de mercado, para ello se debe obtener la rentabilidad y riesgo, conceptos muy importantes, representados por los estadígrafos de retorno medio o esperado, desviación sobre el retorno medio y la covarianza entre los activos los que permite conformar portafolios superiores a los activos individuales.

Luego se ha introducido como activo libre de riesgo para el mercado peruano a los CDBCRP, que ayuda a construir la línea de portafolios; $y$, posteriormente, al optimizar las proporciones de activos en la frontera eficiente versus el activo libre de riesgo conseguimos la cartera de mercado con la que procedemos a construir la LMC, de gran importancia ya que es allí donde los porfolio manager llevan a cabo realmente las combinaciones del portafolio de inversiones, obteniendo luego perfiles para distintos tipos de inversionistas, y las AFP clasifican en los fondos de tipos 1, 2 y 3 (conservador, 


\section{Portafolio de inversiones y desempeño del sistema privado de pensiones peruano: 2000 - 2005}

moderado, agresivo).

El análisis de desempeño o performance, según los índices de Sharpe y Treynor, ha servido para calificar a las AFP de acuerdo a su gestión de portafolio, el nivel de rentabilidad obtenido por unidad de riesgo asumido. El Índice de Jensen ha servido para el análisis de selectividad. El Índice de Treynor-Mazuy se utilizó para el análisis de timing.

\section{CONCLUSIONES}

Se evaluó la eficiencia de mercado, el equilibrio de activos financieros, la frontera eficiente y el desempeño de gestión de portafolio para las cuatro AFP del mercado peruano que registran data estadística para el período enero 2000 - diciembre 2005. Para ello, se ha tomado como insumos el valor cuota tradingday, el rendimiento de activos, el índice ISBVL, usado como proxy del portafolio de mercado, los CDBCRP como Proxy del activo libre de riesgo.

De los análisis realizados, se concluye lo siguiente:

1. Mediante el análisis de inversiones, se ha probado la hipótesis que el portafolio de inversiones y comportamiento de las AFP peruanas no están alcanzando niveles óptimos. Sin embargo, sus líneas de portafolio se están acercando.

2. Según los resultados del estudio de eventos, para verificar que tan rápido reaccionan los precios a la liberación de noticias, el mercado en que se desenvuelven las AFP es eficiente, por lo menos en su forma débil.

El análisis de desempeño a través de la metodología de los indicadores de Sharpe y Treynor señala que las AFP obtuvieron rendimientos superiores a los del mercado. Del análisis econométrico, el índice de Jensen evidencia señales de la habilidad de selectividad, en tanto que para el índice de Treynor-Mazuy no existe la habilidad de timing. El ranking elaborado según los indicadores de Sharpe, Treynor y Jensen, para los tres casos coloca a las AFP Integra y Horizonte en primer y segundo lugar. 


\section{David Juan Sanchez Cruz}

\section{BIBLIOGRAFÍA}

Aguilar, Julio y Rodríguez Allan (1998). El Mercado de Capitales en Perú después de la Reforma Económica. CEN 111.

CONASEV (1996 - 2004). Informe Semanal Bursátil.

Húmala, A. (1995). "Gestión de Portafolio y Rentabilidad de los Fondos Mutuos". Gerencia de Estudios Económicos del Banco Central de Reserva del Perú. XII Encuentro de Economistas.

Walker, H. (1993). "Desempeño Financiero de las Carteras Accionarias de los Fondos de Pensiones en Chile. ¿Ha tenido desventajas ser grandes?”. En: Cuadernos de Economía. Año 30, N. ${ }^{\circ}$ 89. Abril de 1993.

Sharpe, W. G. Alexander, y J. Bailey (1995). Investments. 5. ${ }^{a}$ ed., Prentice-Hall, Englewood Cliffs.

Superintendencia de Administradoras de Fondos de Pensiones (1994-1997). Boletín Informativo Semanal.

Superintendencia de Bancos, Seguros y AFP (2000 - 2005). Boletín mensual.

Superintendencia de Bancos, Seguros y AFP (2000 - 2005). Memoria Anual.

Superintendencia de Bancos, Seguros y AFP (2000 - 2005). The New Strengths of Peru. 